\title{
Active vibration isolation experiments using translational and rotational power transmission as a cost function
}

\author{
Carl Q. Howard a) and Colin H. Hansen \\ Active Noise and Vibration Control Group, School of Mechanical Engineering. The University of Adelaide, \\ SA 5005, Australia
}

(Received 28 February 2005; revised 15 May 2006; accepted 25 June 2006)

Active vibration isolation experiments were conducted using a transducer that measures
translational and rotational power transmission from a vibrating mass, through a single-axis active
isolator and into a beam: The transducer is capable of measuring forces and moments along six axes
and an accelerometer array measures its motion. By combining the measured force and velocity
signals the translational and rotational.power transmission was measured. Comparisons were made
of the effectiveness of several cost functions for minimizing the vibration transmitted into the beam.
The results show that active vibration isolation using power transmission as a cost function to be
minimized is limited by the phase accuracy of the transducers. The best results were obtained from
the minimization of the weighted sum of force and velocity. C 2006 Acoustical Society of America. [DOI: 10.1121/1.2228839]

PACS number(s): 43.40.Vn, 43.40.At [KC]

Pages: 2004-2016

\section{INTRODUCTION}

Vibrating machinery usually generates vibration forces in more than one direction and vibration isolators are often used to reduce the transmission of vibration from the machine into the supporting structure. Typically, vibration isolators are selected to maximize vibration attenuation in the predominant vibrating direction, which is often the translational vertical axis. However, previous research has shown that the vibrational power transmission from rotational moments cannot be neglected when considering the total vibrational power transmitted into the receiving structure.

Here, results of an experimental investigation of the active vibration isolation of a vibrating rigid mass from a simply supported beam are presented. The active vibration isolator used for the investigation has a single control actuator which is orientated vertically. The six-axis vibratory power transducer described in Howard ${ }^{1}$ is used to measure the vibratory power transmitted from a vibrating rigid mass, through a vibration isolator, and into the simply supported beam. Several cost functions are compared in terms of their effectiveness at reducing the vibration transmitted into the simply supported beam. The cost functions that are compared are various combinations of squared translational and rotational accelerations, the weighted sum of squared translational force and velocity and rotational moments and velocities, signed translational and rotational power transmission, and squared translational and rotational power transmission. Predictions of the vibration isolation attenuation are made using the theory described in Howard. 'The predictions are compared with experimental measurements of the active vibration isolation performance using a singleaxis active vibration isolator.

The novel work presented in this paper is the active vibration isolation experiments involving the minimization

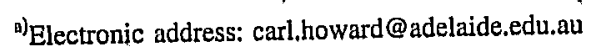

of cost functions that include translational forces and rotational moments, and translational and rotational power transmission. The reason why this work has not been attempted previously is because of the lack of suitable transducers capable of measuring power transmission by moments. The results from experiments presented here provide experimental evidence to support previously published theoretical predictions on the power circulation (or negative power flow) phenomenon that can occur in active vibration isolation transmission is omitted. In addition, the experimental results demonstrate that the phase errors in the transducers used to transmission as a cost function to be minimized. A better cost function, which is not sensitive to phase inaccuracies, is the weighted sum of the squared translational forces and velocities, and rotational moments and rotational velocities.

\section{PREVIOUS WORK}

Active vibration isolation experiments have been conducted by several researchers. ${ }^{2-5}$ However, in almost all previous work they have neglected the contribution and measurement of power transmission by moments, because suitable transducers were not available. Instead, researchers have attempted to indirectly estimate the vibrational power flowing through the support structure rather than directly measuring the vibrational power flowing into the support structure. Pinnington. ${ }^{6}$ considered the power transmitted from passive isolators was measured using two techniques which did not require the measurement of force at the bottom of the which measured the force and acceleration at the mounting point of each isolator. The first practical method of measuring power transmission through each isolator was to measure implementations when the contribution of rotational power measure power transmission limit the usefulness of power a machine into à longitudinally stiffened plate, using a multipole expansion technique. Power transmission through four isolator, and was compared with a reference technique, 
the source acceleration. The second method was to estimate the magnitude of power generated by all sources of vibration, including airborne noise. It was shown that the two measurement techniques agreed with the reference technique.

Gardonio et $a l^{7,8}$ theoretically examined the power transmission of a vibrating rigid mass isolated from a plate using two active mounts. They showed that minimization of the out-of-plane component of power, when power transmission due to moments was omitted, caused a "power circulation" phenomena (see also Refs. 9 and 10), where power was drawn into the support plate and then reabsorbed by the active mounts. Power circulation caused greater vibration levels in the plate than without active control. Gardonio's work used two different types of cost function. The first was the out-of-plane power transmission, which was capable of negative values and the second was the weighted sum of the out-of-plane squared velocity and squared force, which is positive definite. A weighting factor was applied to the squared force error signal so that it was the same order of magnitude as the squared velocity signal. In this case, the weighting factor was chosen to be the square of the point mobility of the receiving structure. Gardonio et al. reported that the second cost function gave better results than the first. This result is not surprising as the second cost function is always positive and by the definition of power transmission, if the squared velocity or squared force is reduced to zero, then the power transmission along a vertical (out-of-plane) axis is also reduced to zero. The surprising result was that the second cost function gave results close to the minimization of total power transmission, except at a few frequencies where active plus passive isolation was worse than just passive isolation.

Although active vibration isolators have been considered in the past, previous authors have used vibration amplitude squared as the cost function, which does not necessarily relate to the power transmission into the support structure. ${ }^{11,12}$ Work which deals with the active vibration isolation of machinery from flexible supports, which uses the power transmitted into the structure as the cost function to be minimized, has also been reported. ${ }^{2,4}$ In this work, the power transmission was optimized by manual adjustment of the control forces to minimize the product of force and velocity. However, only the power transmission along a single translational axis was considered, whereas previous research ${ }^{13-18}$ has shown the importance of considering power transmission from both translational and rotation axes.

Moorhouse ${ }^{19}$ discusses theoretical aspects of the relative importance of force and moment loading on several structural systems such as finite and infinite plates. The methods can be used to identify potential locations for active vibration control sources on a structure.

Ji et al..$^{20}$ describe a numerical "power mode" approach to estimate power transmission from forces and moments. Their concept is similar to the "radiation mode" approach that is often used in active noise control analyses.

Royston and Singh ${ }^{21}$ have considered the active isolation of a vibrating rigid body from a simply supported beam which used a nonlinear spring as a passive vibration isolating element and an "active force input" as a control actuator to cancel the primary disturbance. The active force input was aligned in the vertical axis with the spring and excitation force. Royston and Singh neglected any rotational or horizontal motion because of the difficulty in measuring the rotational dynamics of the system, but noted in the literature review that power transmission by rotational motion was considered important by previous authors.

While there are many theoretical studies that highlight the importance of rotational power transmission in the measurement of total power transmission, few researchers conduct experimental measurements of rotational power transmission.

To measure the power transmission along six axes (three rotational and three translational), a unique "impedance head" is needed to measure the force and acceleration in each of these directions. Although previous authors have considered multiple axis vibration isolators, for use in the aerospace industry ${ }^{22-25}$ and machinery vibration isolation, ${ }^{26-28}$ there has not been any experimental work reported that uses an active vibration isolator to minimize both translational and rotational vibration. Although Sanderson ${ }^{29}$ has measured moment mobilities in structures, there has not been any experimental work in active vibration isolation which minimizes the transmission of rotational moment loads.

Howard et al. ${ }^{9}$ showed that passive plus active vibration isolation, using vibrational power along a vertical axis as the cost function to be minimized, can increase the vibrational power transmission into the support structure compared with just passive isolation. A similar problem has been examined by Gardonio et al. ${ }^{8}$ for a plate, but the problem has not been examined for a beam or a cylinder.

This paper presents results from active vibration isolation experiments where translational force and accelerations as well as rotational moments and accelerations are used as cost functions to be minimized. Experimental results showing the active vibration isolation performance are derived from measured transfer function data, which is described in the next section.

\section{TRANSFER FUNCTION METHOD TO PREDICT THE VIBRATION ISOLATION USING ACTIVE CONTROL}

The method used here to predict the isolation performance of the system uses measured transfer function data. A similar method has been used by Dorling et $a l^{30,31}$ where measured acoustic transfer function data were used to predict the sound-pressure levels inside an aircraft cabin as a result of active noise control.

Transfer functions were measured between the driving force on the structure and the response at the error sensors. The driving force was measured by placing a force transducer between a primary shaker and the structure. Response measurements were made at the six-axis force transducer and the acceleration transducers. Transfer functions were also measured between the primary shaker and the error sensors and between the control shaker and the error sensors.

The error signals from the error sensors can be written in matrix form as [Ref. 32, Appendix A.5], 


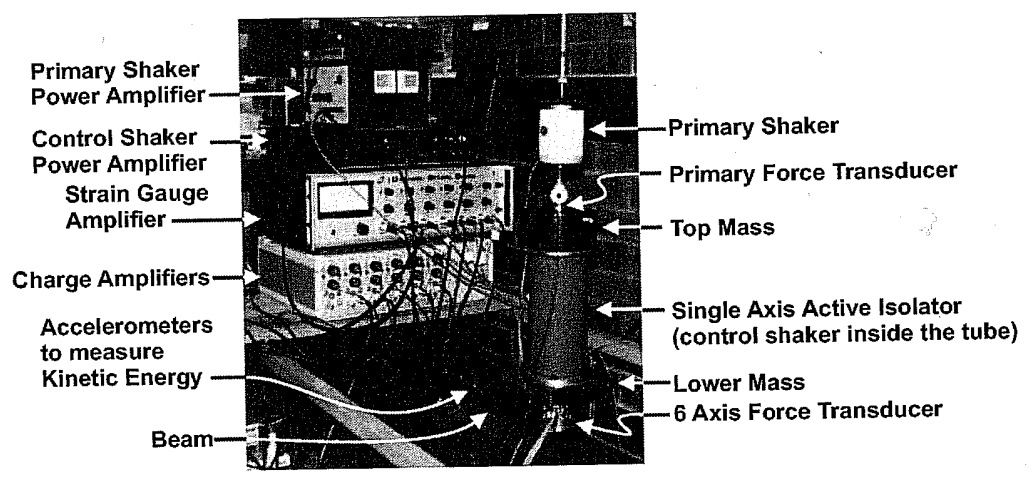

FIG. 1. (Color online) Experimental setup for the single axis isolator on the simply supported beam.

$$
\mathbf{e}=\mathbf{d}+\mathbf{C x}
$$

where $\mathbf{e}$ is an $\left(n_{e} \times 1\right)$ vector of $n_{e}$ error signals, $\mathbf{x}$ is a $\left(n_{c}\right.$ $\times 1)$ vector of control signals, $\mathbf{d}$ is an $\left(n_{e} \times 1\right)$ vector of the error signals resulting from passive control, and $\mathbf{C}$ is a $\left(n_{e}\right.$ $\times n_{c}$ ) matrix of the transfer functions between the control signals and the error signals when the primary disturbance is turned off. The usual goal of active control systems is to determine the amplitude and phase of the control signals which will cancel the primary disturbance, and is given by rearrangement of Eq. (1) as

$$
\mathbf{x}_{0}=-(\mathbf{C})^{-1} \mathbf{d}
$$

Equation (2) can be solved when there are an equal number of control signals and error signals $\left(n_{c}=n_{e}\right)$. If there are more error signals than control signals $\left(n_{e}>n_{c}\right)$ then the problem is said to be overdetermined. The matrix $\mathbf{C}$ is not square and cannot be inverted, and generally it is not possible to achieve complete cancellation at all of the error sensors. The problem can be transformed into a least-squares problem such that the cost function $J$ which is minimized is the squared amplitude of the error signals e, which can be written as

$$
\begin{aligned}
J & =\mathbf{e}^{\mathrm{H}} \mathbf{e} \\
& =\mathbf{x}^{\mathrm{H}} \mathbf{C}^{\mathrm{H}} \mathbf{C x}+\mathbf{x}^{\mathrm{H}} \mathbf{C}^{\mathrm{H}} \mathbf{d}+\mathbf{d}^{\mathrm{H}} \mathbf{C x}+\mathbf{d}^{\mathrm{H}} \mathbf{d} .
\end{aligned}
$$

Equation (4) is in the general Hermitian quadratic form, and has a minimum value when the control signals are given by

$$
\mathbf{x}_{0}=-\left(\mathbf{C}^{\mathrm{H}} \mathbf{C}\right)^{-1}\left(\mathbf{C}^{\mathrm{H}} \mathbf{d}\right)
$$

When there are more control sources than error sensors $\left(n_{c}>n_{e}\right)$, the minimization problem becomes underdetermined and there are an infinite number of solutions for the control sources which will minimize the error signals. The problem can be redefined to include a control effort term, such as $\mathbf{x}^{\mathrm{H}} \mathbf{x}$, so that the cost function $J$ is minimized with the least amount of control effort. The cost function $J$ is minimized when the control source is given by

$$
\mathbf{x}_{0}=-\mathbf{C}^{\mathrm{H}}\left(\mathbf{C} \mathbf{C}^{\mathrm{H}}\right)^{-1} \mathbf{d} \text {. }
$$

Consider the system shown in Fig. 1, where the velocity along the vertical axis at the connection between the six-axis force transducer and the beam is to be minimized when the top rigid body is subjected to a harmonic vertical primary force. A transfer function measurement is taken over the frequency range of interest, between the primary driving force and the velocity along the vertical axis at the base of the isolator and this transfer function is called $\mathbf{Z}_{v p}$. The primary driving force is then turned off and a transfer function measurement is taken between the force exerted by the control shaker and the velocity along the vertical axis at the base of the isolator; this transfer function is called $\mathbf{Z}_{v c}$. The terms $\mathbf{d}$ and $\mathbf{C}$ become

$$
\begin{aligned}
& \mathbf{d}=\mathbf{Z}_{v p} \mathbf{f}_{p}, \\
& \mathbf{C}=\mathbf{Z}_{v c},
\end{aligned}
$$

where $\mathbf{f}_{p}$ is the $\left(n_{p} \times 1\right)$ column vector of primary forces, which for this example is $\mathbf{f}_{p}=1$.

In the experiments that follow, the optimal control forces are calculated by using Eq. (5) and (6) depending on the number of error sensors and control forces. In the experiments where signed power transmission is minimized, the optimal control forces are calculated by a similar method, which is explained later in this section.

Gardonio et al. suggested minimizing the weighted sum of squared velocity and squared force along the vertical axis to actively control vibration transmission through an active isolator. They gave the vector of optimal control forces as

$$
\mathbf{x}_{0}=-(\mathbf{A})^{-1} \mathbf{b}
$$

where

$$
\begin{aligned}
& \mathbf{A}=\mathbf{Z}_{v c}^{\mathrm{H}} \mathbf{Z}_{v c}+\mu \mathbf{Z}_{f c}^{\mathrm{H}} \mathbf{Z}_{f c}, \\
& \mathbf{b}=\mathbf{Z}_{v c}^{\mathrm{H}} \mathbf{Z}_{v p} \mathbf{f}_{p}+\mu \mathbf{Z}_{f c}^{\mathrm{H}} \mathbf{Z}_{v p} \mathbf{Z}_{f p} \mathbf{f}_{p},
\end{aligned}
$$

where $\mu$ is the weighting factor which is applied to the squared force signal so that the amplitudes of the squared velocity signals and squared force signals are similar, $\mathbf{Z}_{i j}$ is a transfer function between velocity or force, $i$, and primary or control force, $j$. For example, $\mathbf{Z}_{v c}$ is the transfer function matrix of dimensions $\left(n_{e} \times n_{c}\right)$ between the velocity measured at an error sensor and the driving control force.

When there are more error sensors than control forces, Eqs. (9) and (11) presented in Ref. 8 cannot be solved and have to be rewritten in terms of the least-squares problem formulation. The velocities and forces at the error sensors can be written as

$$
\begin{aligned}
& \mathbf{v}=\mathbf{Z}_{v p} \mathbf{f}_{p}+\mathbf{Z}_{v c} \mathbf{f}_{c} \\
& \mathbf{f}=\mathbf{Z}_{f p} \mathbf{f}_{p}+\mathbf{Z}_{f c} \mathbf{f}_{c} .
\end{aligned}
$$

The terms $\mathbf{d}$ and $\mathbf{C}$ become 


$$
\begin{aligned}
& \mathbf{d}=\left[\begin{array}{c}
\mathbf{Z}_{v p} \mathbf{f}_{p} \\
\sqrt{\mu} \mathbf{Z}_{f p} \mathbf{f}_{p}
\end{array}\right] \\
& \mathbf{C}=\left[\begin{array}{c}
\mathbf{Z}_{v c} \\
\sqrt{\mu} \mathbf{Z}_{f c}
\end{array}\right] .
\end{aligned}
$$

Equation (5) and (6) can now be used to calculate the optimal control forces depending on the number of error sensors and control forces.

The method described here can always be used to calculate the theoretical control force that will minimize the theoretical cost function based on measured transfer function data. However, whether the control force can actually be implemented in practice depends on the primary disturbance and the causality of the transfer functions and the causality of Eqs. (5) and (6). If the primary disturbance is tonal (periodic), then the causality issues are not of concern. However, if the primary disturbance is unpredictable (random), then the causality issues are important. The causality issues are further discussed in Ref. 32, (Chap. 8.6).

The calculation of the optimum control forces for the minimization of signed power transmission can be derived in a similar manner as the previous derivation. The velocity and force at the $n_{e}$ error sensors can be described by vectors $\mathbf{v}_{t}$ and $\mathbf{f}_{t}$ which have length $n_{e}$. The velocity and force vectors are given by

$$
\begin{aligned}
& \mathbf{v}_{t}=\mathbf{Z}_{v p} \mathbf{f}_{p}+\mathbf{Z}_{v c} \mathbf{f}_{c}, \\
& \mathbf{f}_{t}=\mathbf{Z}_{f p} \mathbf{f}_{p}+\mathbf{Z}_{f c} \mathbf{f}_{c},
\end{aligned}
$$

where $\mathbf{f}_{p}$ and $\mathbf{f}_{c}$ are the primary and control force column vectors of length $n_{p}$ and $n_{c}$, respectively, $\mathbf{Z}_{i j}$ is a transfer function between velocity or force, $i$, and primary or control force, $j$. For example, $\mathbf{Z}_{f c}$ is the transfer function matrix of dimensions $\left(n_{e} \times n_{c}\right)$ between the forces measured at the error sensors and the driving control force. These definitions can be used to define the time-averaged harmonic vibrational power transmission into the structure as

$$
\text { Power }=\frac{1}{2} \operatorname{Re}\left(\mathbf{v}_{t}^{\mathrm{H}} \mathbf{f}_{t}\right)
$$

where the superscript $H$ is the Hermitian transpose. Substitution of Eqs. (16) and (17) into Eq. (18) and rearranging results in a quadratic expression in terms of the control force $\mathbf{q}_{c}$

$$
\text { Power }=\frac{1}{2}\left(\mathbf{q}_{c}^{\mathrm{H}} \boldsymbol{\alpha} \mathbf{q}_{c}+\mathbf{q}_{c}^{\mathrm{H}} \boldsymbol{\beta}+\boldsymbol{\beta}^{\mathrm{H}} \mathbf{q}_{c}+c^{i}\right)
$$

where

$$
\begin{aligned}
& \mathbf{q}_{c}=\left[\begin{array}{c}
\mathbf{f}_{c}^{r} \\
\mathbf{f}_{c}^{i}
\end{array}\right] \\
& \boldsymbol{\alpha}=\boldsymbol{\alpha}^{T}=\frac{1}{2}\left[\begin{array}{cc}
\mathbf{a}^{i}+\left(\mathbf{a}^{i}\right)^{T} & \mathbf{a}^{r}-\left(\mathbf{a}^{r}\right)^{T} \\
-\mathbf{a}^{r}+\left(\mathbf{a}^{r}\right)^{T} & \mathbf{a}^{i}+\left(\mathbf{a}^{i}\right)^{T}
\end{array}\right]
\end{aligned}
$$

$$
\boldsymbol{\beta}=\frac{1}{2}\left[\begin{array}{c}
\left(\mathbf{b}_{2}^{i}\right)^{T}+\mathbf{b}_{1}^{i} \\
\left(\mathbf{b}_{2}^{r}\right)^{T}-\mathbf{b}_{1}^{r}
\end{array}\right],
$$

and the real matrices $\mathbf{f}_{c}^{r}, \mathbf{f}_{c}^{i}, \mathbf{a}^{r}, \mathbf{a}^{i}, \mathbf{b}_{1}^{r}, \ldots$ represent, respectively, the real and imaginary parts of the complex matrices $\mathbf{f}_{c}, \mathbf{a}, \mathbf{b}_{1}$, and $\mathbf{b}_{2}$ and the complex constant $c$, which are defined as

$$
\begin{aligned}
& \mathbf{a}=\mathbf{Z}_{v c}^{\mathrm{H}} \mathbf{Z}_{f c}, \\
& \mathbf{b}_{1}=\mathbf{Z}_{v c}^{\mathrm{H}} \mathbf{Z}_{f p} \mathbf{f}_{p}, \\
& \mathbf{b}_{2}=\mathbf{f}_{p}^{\mathrm{H}} \mathbf{Z}_{v p}^{\mathrm{H}} \mathbf{Z}_{f c}, \\
& c=\mathbf{f}_{p}^{\mathrm{H}} \mathbf{Z}_{v p}^{\mathrm{H}} \mathbf{Z}_{f p} \mathbf{f}_{p} .
\end{aligned}
$$

The power transmission into the system for passive vibration isolation $\left(\mathbf{q}_{c}=[0,0]^{\mathrm{T}}\right)$ is given by $c^{i} / 2$. The minimum of Eq. (19) is given by

$$
\text { Power }_{\min }=-\frac{1}{2}\left(\boldsymbol{\beta}^{\mathrm{T}} \boldsymbol{\alpha}^{-1} \boldsymbol{\beta}+c^{i}\right)
$$

corresponding to an optimum control force vector given by

$$
\left(\mathbf{q}_{c}\right)_{\mathrm{opt}}=-\boldsymbol{\alpha}^{-1} \boldsymbol{\beta} \text {. }
$$

The derivation of the optimum control forces to minimize squared power transmission can be achieved by following the same process as above and has not been included here. Readers are referred to Howard ${ }^{1}$ for a complete derivation.

\section{DESCRIPTION OF THE EXPERIMENTAL SETUP}

Figure 1 shows a picture of the experimental rig and Fig. 2 shows how the instruments were connected. A steel beam, of dimensions $1.55 \mathrm{~m}$ long by $25 \mathrm{~mm}$ square, was mounted between two knife edges which provided simply supported end conditions. The six-axis force transducer, described in Howard, ${ }^{1}$ was bolted to the beam, $0.75 \mathrm{~m}$ from the end of the beam. Attached to the top of the force transducer was the lower mass, which was used to support the end of the vibration isolator. The vibration isolator was a cylindrical polyurethane tube and inside the tube was a Ling Dynamics V203 shaker which provided a canceling force to counteract the vibrations which passed through the outer tube. On top of the vibration isolator was a solid-steel cylindrical mass which weighed $7.4 \mathrm{~kg}$. The control shaker was connected to the rigid mass with a "stinger" that is stiff along the axis of the shaker but is rotationally flexible. Five accelerometers were attached to the beam to measure its residual vibration when active control was applied. The five accelerometers were used to measure the velocity of the beam and were mounted at $0.30,0.35,0.40,0.45$, and $0.50 \mathrm{~m}$ from its end. For the experimental system considered here, it was found that there were three vibrational axes of greatest importance for power transmission: vertical translation and two rotational axes which did not include the drilling axis through the isolator. Four accelerometers were attached to the six-axis force transducer and used to calculate the acceleration of the material beneath the strain gauges, using the method described in Howard. ${ }^{1}$ The use of four accelerometers enables the mea- 


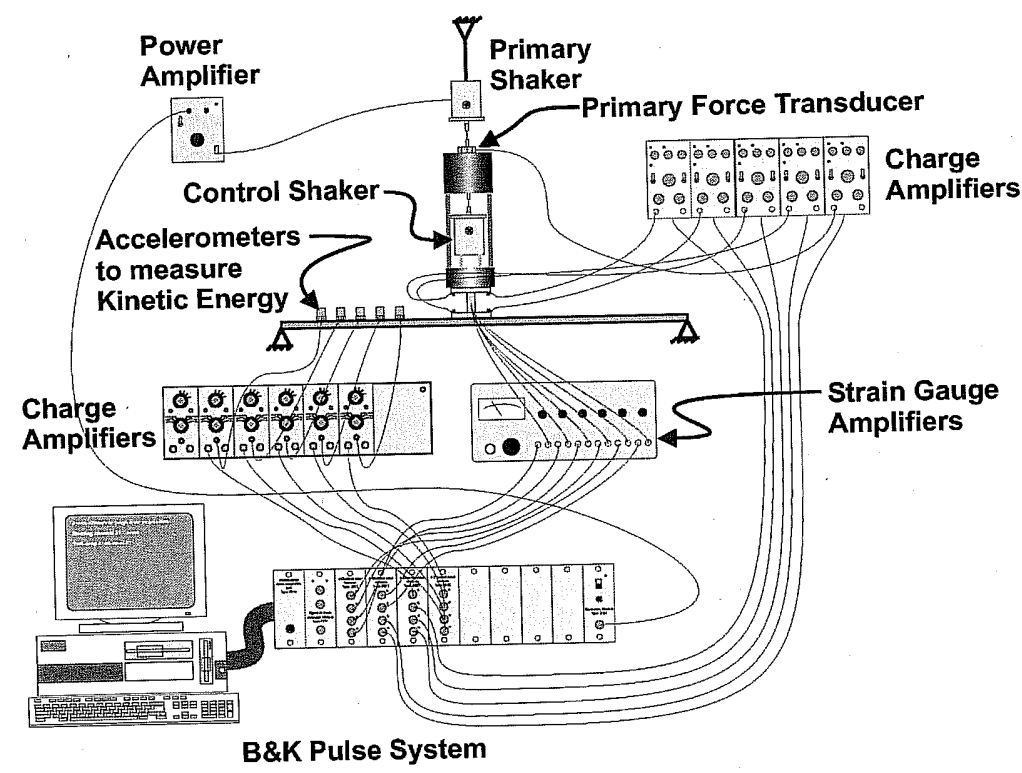

FIG. 2. (Color online) Setup of the instruments for the experiment of the single axis isolator and the simply supported beam.

surement of translational motion and of rotational about one axis. These four accelerometers were repositioned so that motion about a second rotational axis could be measured.

Figure 2 shows how the instrumentation was connected. All the transducers were connected to amplifiers which were connected to the Brïel \& Kjær Pulse System, which in turn measured the transfer functions. The primary shaker was connected to the top mass through a B\&K force transducer, which was used to measure the primary translational force, and applied a harmonic force which swept in frequency between $5 \mathrm{~Hz}$ and $200 \mathrm{~Hz}$.

The vibration isolation performance described here is quantified by the change in the average of the squared velocity of the beam measured using five accelerometers. The average of the squared velocity of the beam is proportional to the kinetic-energy (KE) of the beam. The true value of the $\mathrm{KE}$ is calculated by the summation of an infinite number of squared velocity measurements over the length of the beam to measure the translational and rotational accelerations, multiplied by the mass of the beam, and has units of joules. The metric used to describe the relative reduction in beam vibration in the experimental results presented here is proportional to the $\mathrm{KE}$, as the mass term has been neglected, and a finite number of accelerometers was used. This measurement is not affected by phase errors and provides a reasonable approximation of the global $\mathrm{KE}$ of the beam. It also provides an independent measure of the isolation performance. Comparisons of the isolation performance using a single sensor, for example the acceleration at the base of the isolator, do not provide a good measure because it is possible to minimize the vibration at the sensor and increase the vibration elsewhere on the supporting structure. Some of the theoretical predictions and experimental results to follow are limited in validity as only five accelerometers were used to measure the average of the squared velocity. This limitation is addressed when it is apparent that it affects the results.

The physical properties of the simply supported beam and isolator system are shown in Table I and are used with the theoretical analysis presented in Howard. ${ }^{1}$ The resonance frequencies of the simply supported beam, without the isolator attached, were measured to be 29, 103, and $234 \mathrm{~Hz}$.

\section{EXPERIMENTAL RESULTS}

In this section, the vibration isolation performance of an active vibration isolation system when various cost functions are minimized is compared with and without active control. It should be noted that no attempt was made to optimize the design of the polyurethane tube that provided passive vibration isolation. It is possible to obtain much higher vibration isolation using a properly designed passive vibration isolator than the results presented here. The isolation performance is measured by monitoring the average of the squared velocity of the beam. The transfer function method, which was described in Sec. III, was used to calculate the cost functions and the average of the squared velocity of the beam.

\section{A. No control}

Figure 3 shows that the experimentally measured values of power transmission into the beam do not match the theoretically predicted values. The difference is attributed to the phase errors in the transducers. The phase accuracy of the force transducer and accelerometer combination was measured to be about $\pm 2^{\circ} .{ }^{1}$ Figure 4 shows that there is a random $\pm 2^{\circ}$ phase error of the relative phase angle between force and displacement for theory and experiment. The difference in phase angles between force and displacement is close to $180^{\circ}$, which means that the difference between the force and velocity would be very close to $90^{\circ}$, and hence the small

TABLE I. Parameters used in the active isolator and beam system.

\begin{tabular}{lccc}
\hline \hline Beam length & $1.550 \mathrm{~m}$ & Beamwidth & $0.025 \mathrm{~m}$ \\
Beam thickness & $0.025 \mathrm{~m}$ & Isolator location & $0.750 \mathrm{~m}$ \\
Young's modulus & $207 \mathrm{GPa}$ & Moment of inertia & $1.6 \times 10^{-5} \mathrm{~m}^{4}$ \\
Beam density & $7800 \mathrm{~kg} / \mathrm{m}^{3}$ & Beam damping & $7.48 \times 10^{-6} \mathrm{sN} / \mathrm{m}$ \\
Isolator stiffness $k_{z}$ & $45870 \mathrm{~N} / \mathrm{m}$ & Isolator damping $c_{z}$ & $140 \mathrm{sN} / \mathrm{m}$ \\
Isolator stiffness $k_{\theta_{y}}$ & $216 \mathrm{~N} / \mathrm{rad}$ & Isolator damping $c_{\theta_{y}}$ & $140 \mathrm{sN} / \mathrm{rad}$ \\
Top mass & $7.4 \mathrm{~kg}$ & Bottom mass & $8.2 \mathrm{~kg}$ \\
\hline
\end{tabular}




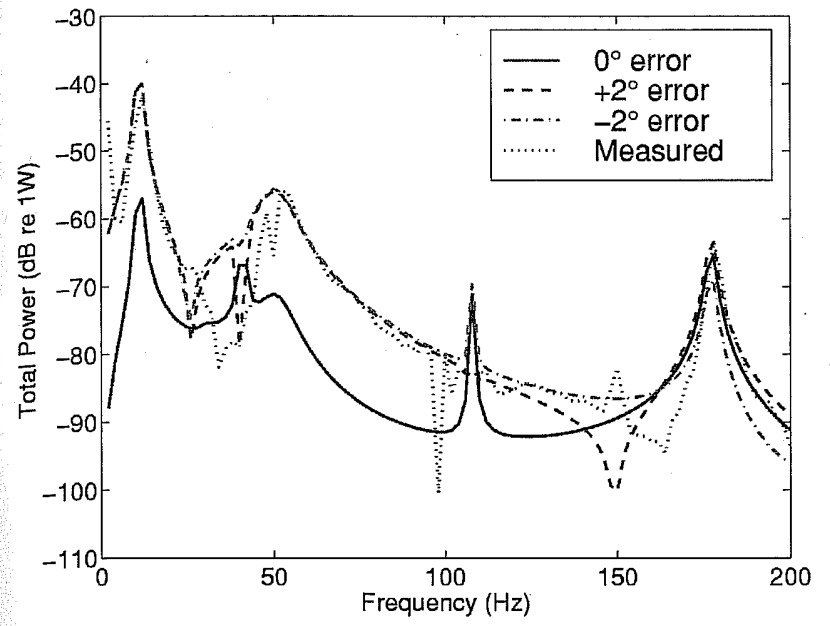

FIG. 3. (Color online) Theoretically predicted and experimentally measured power transmission along the vertical $Z$ axis for a vertical primary force of $F_{z}=1 \mathrm{~N}$. The theoretical values of power transmission are shown for $0^{\circ}$, $+2^{\circ}$, and $-2^{\circ}$ phase errors.

errors in the phase measurements have led to the erroneous measurements of power transmission. Theoretical predictions of the total power transmission were made when there was an artificially imposed error in the relative phase between force and velocity of $\pm 2^{\circ}$ and $-2^{\circ}$, and the results are shown in Fig. 3. The results show that the theoretical predictions with the imposed phase errors appear similar to the experimentally measured results for power transmission.

\section{B. Active control}

Theoretical predictions and experimental results are presented for the cases of with and without active isolation of a vibrating rigid mass that is actively isolated from the beam. It is theoretically possible to stop the vibration from the rigid mass from reaching the simply supported beam if the primary force is exactly aligned with the control actuator. In reality, this is difficult to achieve as there is usually a small misalignment between the primary shaker and the centroid of the rigid mass. For the theoretical results presented in this

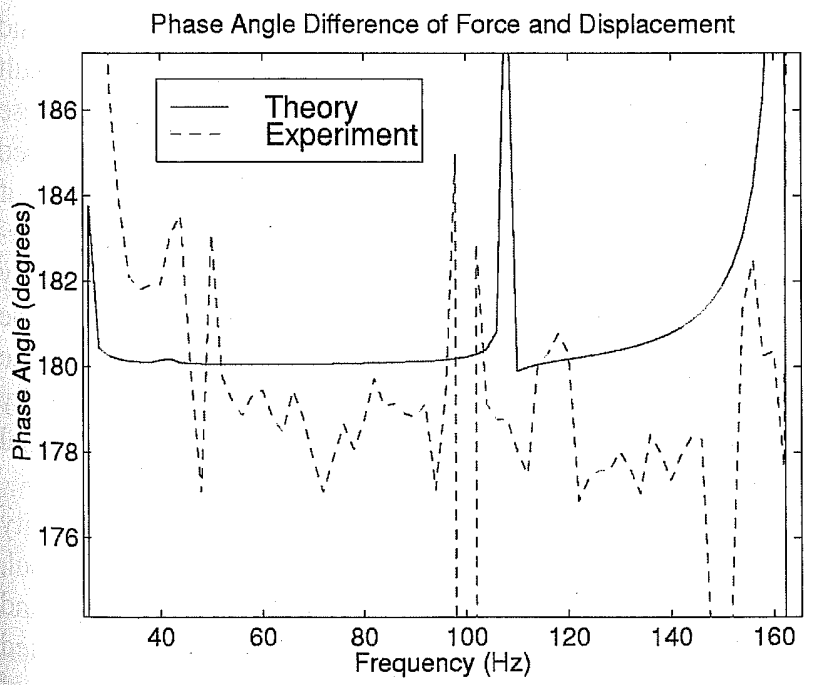

FIG. 4. (Color online) Theoretically predicted and experimentally measured phase angle between force and displacement corresponding to the same configuration shown as shown in Fig. 3.

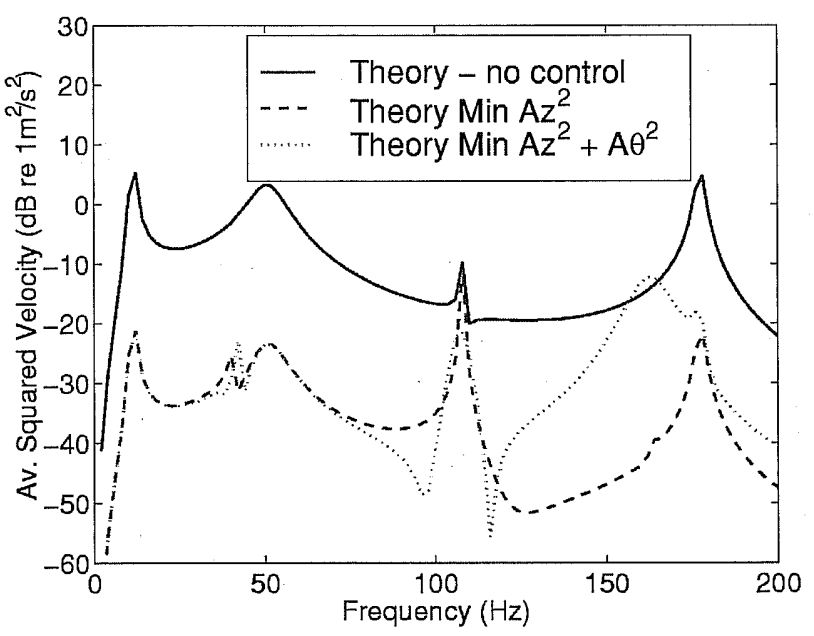

FIG. 5. (Color online) Theoretically predicted average of the squared velocity of the beam for no control, minimization of squared acceleration $A_{z}^{2}$ along the vertical axis and the minimization of the sum of squared accelerations $A_{z}^{2}+A_{\theta y}^{2}$ along the vertical and rotational axes.

section, it is assumed that there is $2 \mathrm{~mm}$ of misalignment, so that the primary load on the top mass is $F_{z}=1 \mathrm{~N}$ and $M_{y}$ $=0.002 \mathrm{Nm}$.

A reasonable approach to the active vibration isolation of this system is to minimize the squared acceleration along the vertical axis at the base of the isolator. Figure 5 shows the theoretically predicted average of the squared velocity of the beam for no control, minimization of squared acceleration $A_{z}^{2}$ along the vertical axis, and the minimization of the sum of the squared accelerations $A_{z}^{2}+A_{\theta y}^{2}$ along the vertical and rotational axes.

The minimization of the sum of the squared accelerations $A_{z}^{2}+A_{\theta y}^{2}$ along the vertical and rotational axes generally results in smaller reductions in the average of the squared velocity of the beam than the minimization of squared acceleration $A_{z}^{2}$ along the vertical axis. At first glance this result appears to be counterintuitive, as controlling two axes might be expected to produce better results than controlling one axis. However, when minimizing the sum of the squared accelerations $A_{z}^{2}+A_{\theta y}^{2}$ there are two error sensors and one control source so the cost function is overdetermined $\left(n_{e}>n_{c}\right)$. In this case, it is not possible to calculate a control force such that the amplitude of both error signals will equal zero. Instead, the sum of the squared accelerations $A_{z}^{2}+A_{\theta y}^{2}$ along the translational and rotational axes is minimized by increasing the squared acceleration along the vertical axis compared to when minimizing only the squared acceleration $A_{z}^{2}$ along the vertical axis. The one exception where this resulted in better performance was at $108 \mathrm{~Hz}$, where it can be seen in Fig. 5 the reduction in average of the squared velocity of the beam at the rotational resonance is greater when controlling the sum of the squared accelerations along the vertical and rotational axes than when controlling the squared acceleration along the vertical axis.

In control systems which have more error signals than control sources $\left(n_{e}>n_{c}\right)$, it is possible to weight the contributions of each error signal to the overall cost function, by multiplying each error signal by a weighting factor, thus providing a mechanism to optimism the results. A large weight- 


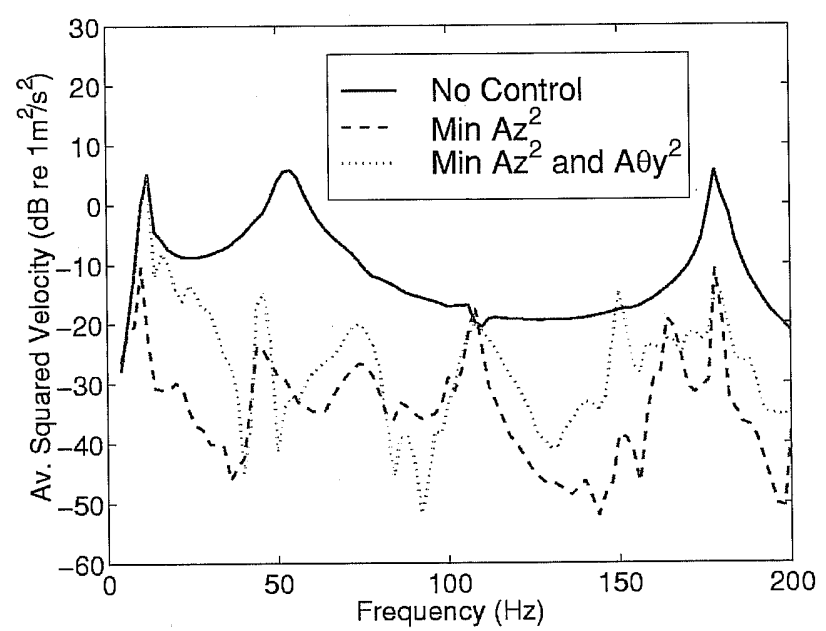

FIG. 6. (Color online) Experimental results for no control, minimization of squared acceleration $A_{z}^{2}$ along the vertical axis and the minimization of the sum of the squared accelerations $A_{z}^{2}+A_{\theta y}^{2}$, along the vertical and rotational axes.

ing factor places greater emphasis on the corresponding error signal in the cost function. Cost functions which use a weighted sum of the error signals are further discussed later in this section.

An experiment was conducted to verify the theoretical predictions shown in Fig. 5 and the results are shown in Fig. 6.

These experimental results confirm the two theoretical predictions that: (1) in general the reduction in the average of the squared velocity of the beam for minimization of the sum of the squared accelerations along the vertical and rotational axes is less than that obtained by minimizing the squared acceleration along the vertical axis; and (2) the reduction in average of the squared velocity of the beam at the rotational resonance is greater when the sum of the squared accelerations along the vertical and rotational axes is minimized than when only the squared acceleration along the vertical axis is minimized.

Figures 5 and 7 show the limitation of using only five

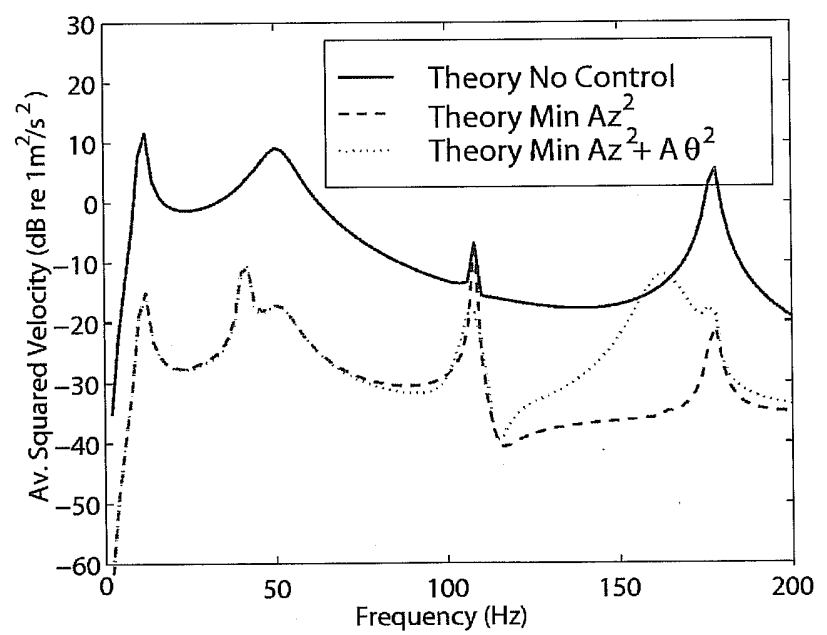

FIG. 7. (Color online) Theoretically predicted average of the squared velocity of the beam (using 14 accelerometers) for no control, minimization of squared acceleration $A_{z}^{2}$ along the vertical axis and the minimization of the sum of the squared accelerations $A_{z}^{2}+A_{\theta y}^{2}$, along the vertical and rotational axes.

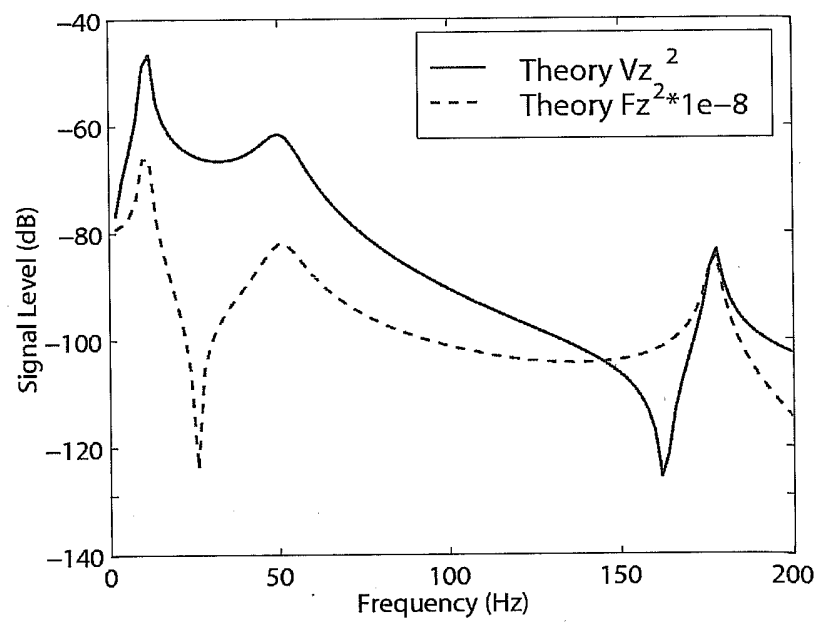

FIG. 8. (Color online) Theoretical signal levels of squared velocity and squared force with the squared force multiplied by a factor of $\mu$ $=10^{-8} \mathrm{~s}^{2} / \mathrm{kg}^{2}$.

accelerometers to measure the average of the squared velocity of the beam. Figure 5 shows that at 95 and $115 \mathrm{~Hz}$ the average of the squared velocity for the minimization of the sum of the squared accelerations along the vertical and rotational axes is lower than that obtained for the minimization of squared acceleration along the vertical axis. Figure 7 shows the same theoretical predictions as Fig. 5, but this time ten accelerometers mounted along the beam and four on the force transducer were used to calculate the average of the squared velocity of the beam. The theoretical results in Fig. 7 show that by using 14 accelerometers to measure the average of the squared velocity of the beam, the isolation performance at 95 and $115 \mathrm{HZ}$ when minimizing the sum of the squared accelerations $A_{z}^{2}+A_{\theta y}^{2}$ along the vertical and rotational axes is similar to minimizing the squared acceleration $A_{z}^{2}$ along the vertical axis. The power transmission spectrum can be related to the KE spectrum by a frequency-dependent function as shown by Pavic.. ${ }^{33}$

\section{Weighted sum of force and velocity}

It has been suggested by Gardonio et al. ${ }^{8}$ that the minimization of a weighted sum of the squared velocity and squared force along the vertical axis will have a similar result to the minimization of total power transmission. The purpose of using a weighted sum of squared velocity and squared force is to adjust the signal levels to be a similar order of magnitude. Figure 8 shows that when the theoretically predicted value of squared force is reduced in amplitude by multiplying by $10^{-8} \mathrm{~s}^{2} / \mathrm{kg}^{2}$ it has a similar signal level to the theoretically predicted squared velocity. Figure 9 shows the corresponding experimentally measured squared velocity signal and the experimentally measured squared force signal multiplied by $\mu=10^{-8} \mathrm{~s}^{2} / \mathrm{kg}^{2}$.

It can be seen in Figs. 8 and 9 that the squared velocity signal level is greater than the weighted squared force signal, except in the frequency range between about 150 and $170 \mathrm{~Hz}$. It is then reasonable to expect that the predicted theoretical and experimental results of the minimization of the weighted sum of squared velocity and squared force should follow the response of the squared velocity except in 


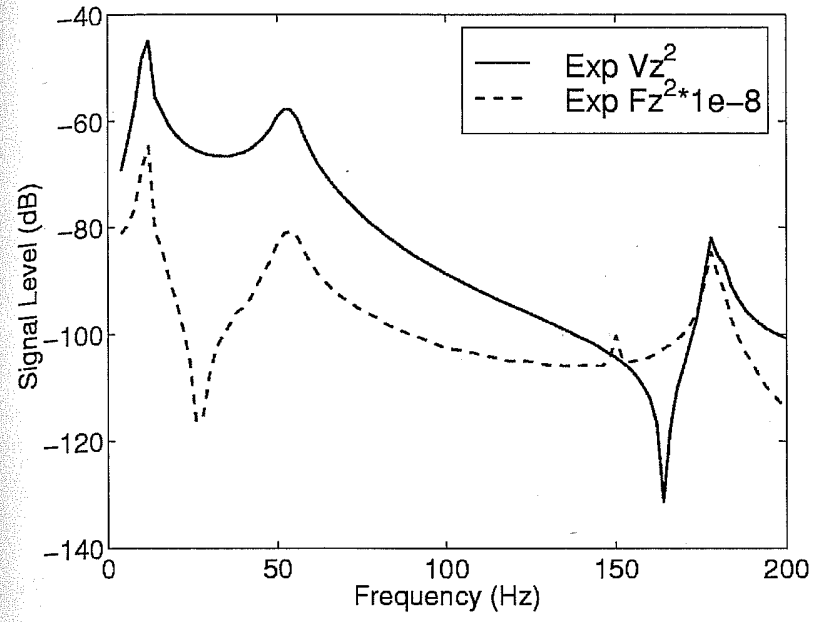

- FIG. 9. (Color online) Experimental results of scaling the squared force signal to be a similar order of magnitude as the squared velocity signal.

the frequency range between 150 and $170 \mathrm{~Hz}$, where is should follow the results for the minimization of the squared force.

Figure 10 shows the theoretically predicted average of the squared velocity of the beam for no control, minimization of squared velocity $V_{z}^{2}$, minimization of squared force $F_{z}^{2}$, and the minimization of the weighted sum of squared velocity and squared force $V_{z}^{2}+\mu F_{z}^{2}$, where $\mu=10^{-8} \mathrm{~s}^{2} / \mathrm{kg}^{2}$.

An experiment was conducted to verify the results from Fig. 10 and the results are shown in Fig. 11. These results show that there is some improvement in results when the minimization of the weighted sum of squared velocity and squared force is used rather than the minimization of squared acceleration.

Gardonio et al. ${ }^{8}$ suggested minimizing the weighted sum of squared velocity and squared force along the vertical axis. Another possibility is to minimize the weighted sum of the squared velocities along translational and rotational axes, squared forces and squared moments. Figure 12 shows the experimentally measured average of the squared velocity of the beam for no control, the minimization of squared veloc-

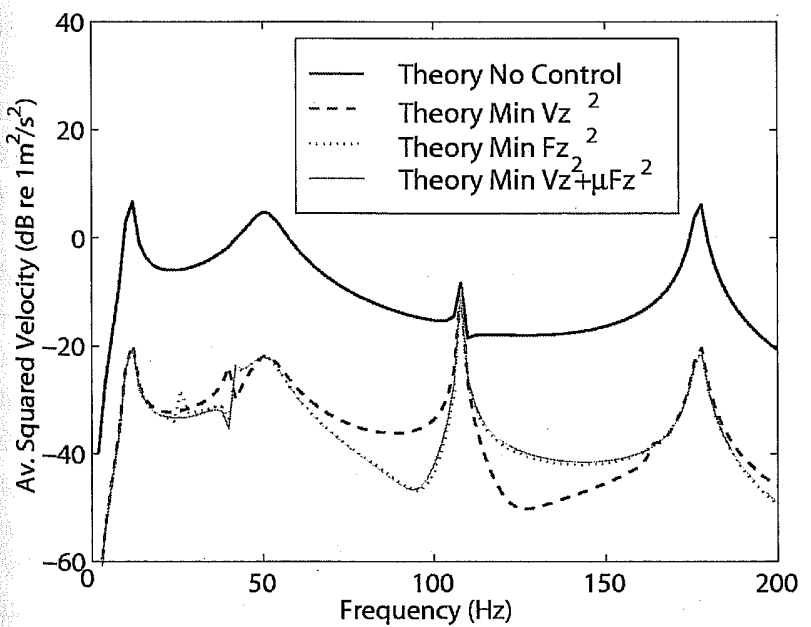

FIG. 10. (Color online) Theoretically predicted average of the squared velocity of the beam for no control, minimization of squared velocity $V_{z}^{2}$ along the vertical axis, minimization of squared force along the vertical axis $F_{z}^{2}$ and the minimization of the weighted sum of $V_{z}^{2}+\mu F_{z}^{2}$ the squared velocity and squared force where $\mu=10^{-8} \mathrm{~s}^{2} / \mathrm{kg}^{2}$.

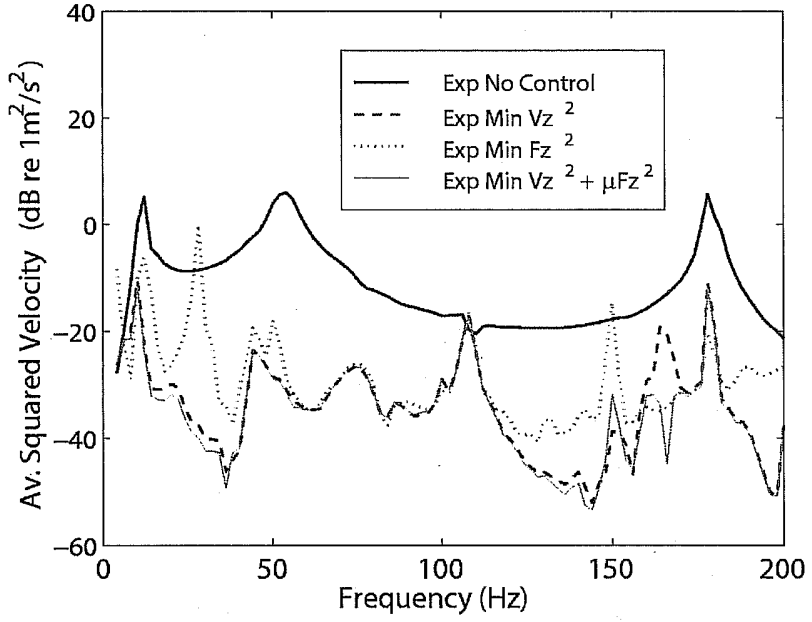

FIG. 11. (Color online) Experimental results of the average of the squared velocity of the beam for no control, minimization of squared velocity along the vertical axis $V_{z}^{2}$, minimization of squared force $F_{z}^{2}$ along the vertical axis and the minimization of the weighted sum of $V_{z}^{2}+\mu F_{z}^{2}$ the squared velocity and squared force.

ity along the vertical axis, and the minimization of the weighted sum of $V_{t}^{2}$ (sum of the squared velocities along the vertical axis $V_{z}^{2}$ and around the rotational axis $V_{\theta y}^{2}$ ) and $F_{t}^{2}$ (the sum of the squared force along the vertical axis $F^{2}$ and the squared moment $M_{y}^{2}$ ). The minimization of $V_{t}^{2}+\mu F_{t}^{2}$, the weighted sum of squared velocities, squared forces, and squared moments along translational and rotational axes, results in slightly better vibration isolation performance than $V_{z}^{2}+\mu F_{z}^{2}$, the weighted sum of the squared velocity and squared force along the vertical axis.

\section{Signed power transmission}

The results from Howard et al. ${ }^{9}$ show that active control using signed power transmission as a cost function to be minimized will converge to a negative value if moments are present and could result in the overall vibration response of the receiving structure being greater than it was with only passive isolation. Signed power transmission is a measure of

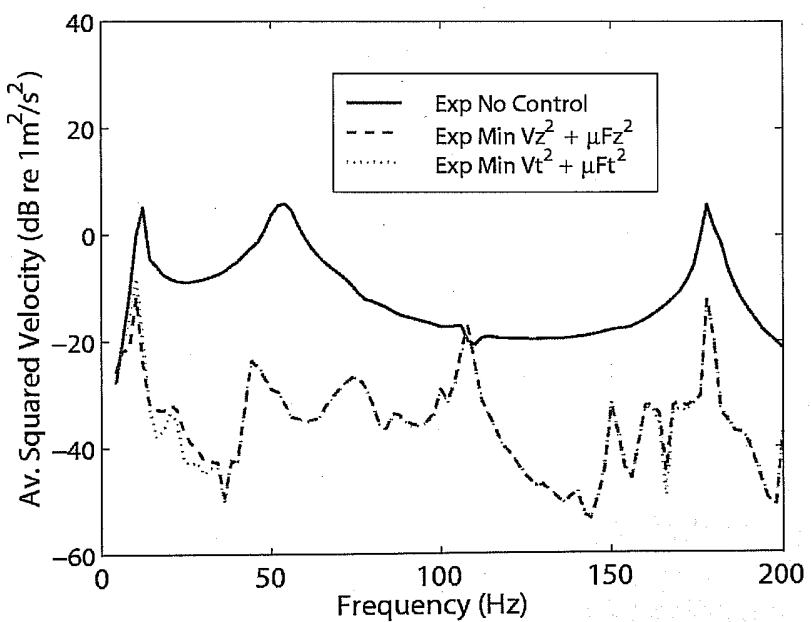

FIG. 12. (Color online) Experimental results of the average of the squared velocity of the beam for no control, minimization of the weighted sum of $V_{z}^{2}+\mu F_{z}^{2}$ the squared velocity and squared force along the vertical axis and the minimization of the weighted sum of $V_{t}^{2}+\mu F_{t}^{2}$, squared velocities, squared forces and squared moments along translational and rotational axes. 


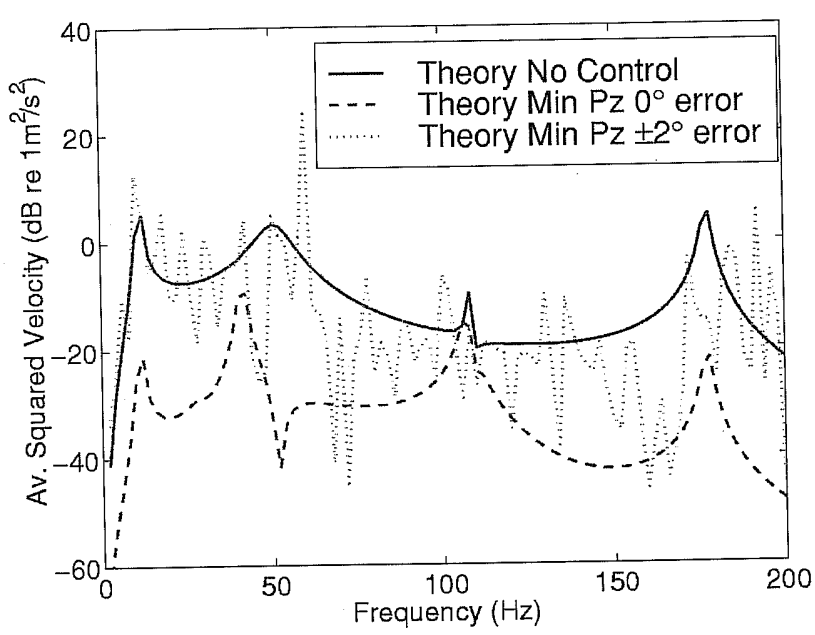

FIG. 13. (Color online) Theoretically predicted average of the squared velocity of the beam for no control, minimization of signed power transmission $P_{z}$ along the vertical axis and when there is $\pm 2^{\circ}$ phase error.

power transmission that takes into account its direction: positive from the vibration source to the support structure and negative for the opposite direction. Minimizing signed power transmission results in the most negative value being optimum. It has been of concern to researchers that small phase errors in the measurement of power transmission can corrupt its true measure such that attempts to reduce vibration transmission using active vibration control, with signed power transmission as a cost function, will be unsatisfactory.

Figure 13 shows a theoretical prediction of the approximated average of the squared velocity of the beam for no control and minimization of signed power transmission and the minimization of signed power transmission when there is a random $\pm 2^{\circ}$ phase error. A phase error between $\pm 2^{\circ}$ was applied to the transfer function measurement between the force response of the structure and the primary load $\mathbf{Z}_{f p}$ and another phase error between $\pm 2^{\circ}$ was applied to the transfer function measurement between the force response of the structure and the force applied by the control actuator $\mathbf{Z}_{f c}$. The use of two different values of phase error for the primary

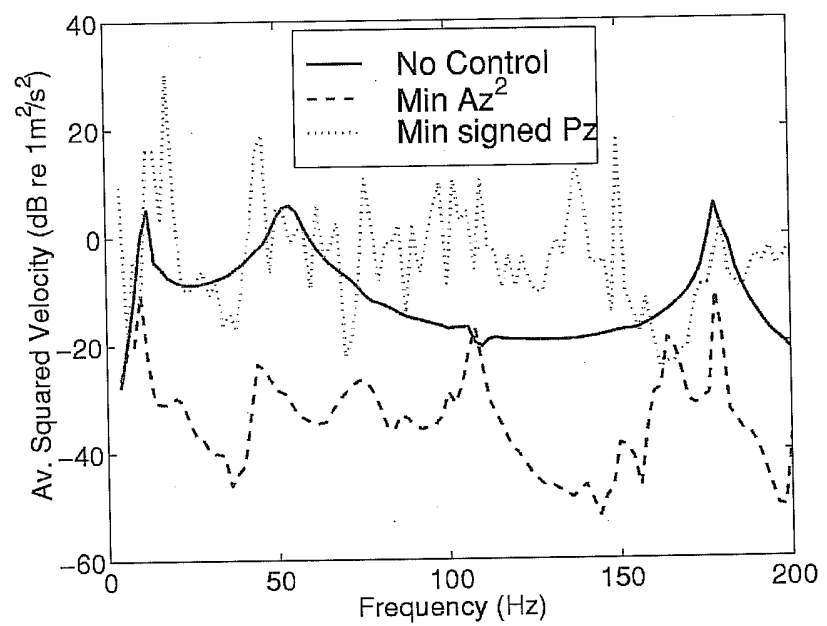

FIG. 14. (Color online) Experimental results of the average of the squared velocity of the beam for no control, minimization of $A_{z}^{2}$ and minimization of the signed power transmission $P_{z}$.

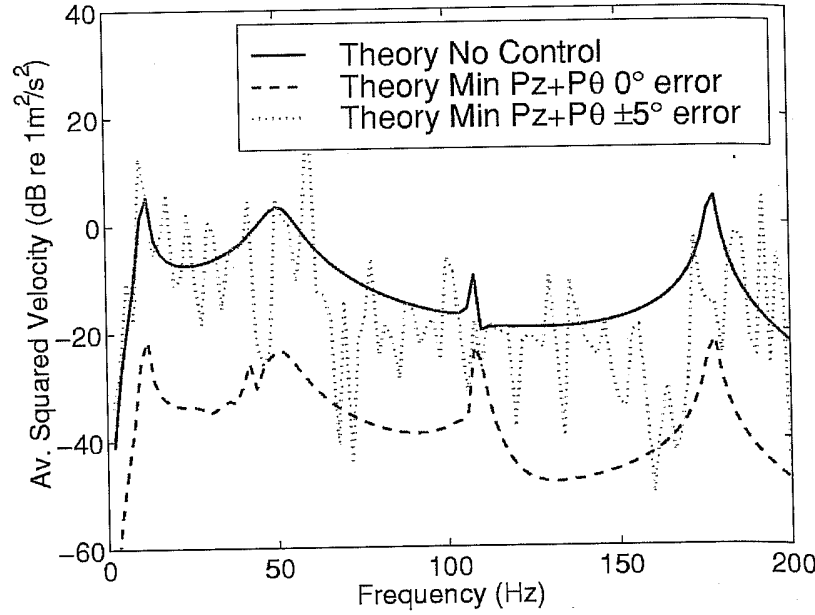

FIG. 15. (Color online) Theoretically predicted average of the squared velocity of the beam for no control, minimization of the sum of $P_{z}+P_{\theta y}$, the signed power transmission along the vertical axes and when there is $\pm 2^{\circ}$ phase error.

and control actuator responses simulates a random phase error that varies with time.

The theoretical result in Fig. 13 shows that the minimization of signed power transmission along a vertical axis with a small phase error will produce unsatisfactory results. This was confirmed in an experiment as shown in Fig. 14.

Similar results occur when the signed total power transmission is minimized. Figure 15 shows the theoretical approximate average of the squared velocity of the beam when the signed power transmission along both the vertical axis and the rotational axis are minimized for an accurate measurement of power and when there is $a \pm 2^{\circ}$ phase error in the measurement of force. Figure 16 shows the corresponding experimental result.

The results presented in Figs. 13-16 verify that attempts to minimize signed power transmission along either a verti$\mathrm{cal}$ axis or along the sum of the vertical and rotational axes will be limited by the phase accuracy of the transducers. This result agrees with the comments by Henriksen ${ }^{34}$ and Gardonio et $a l^{8}$

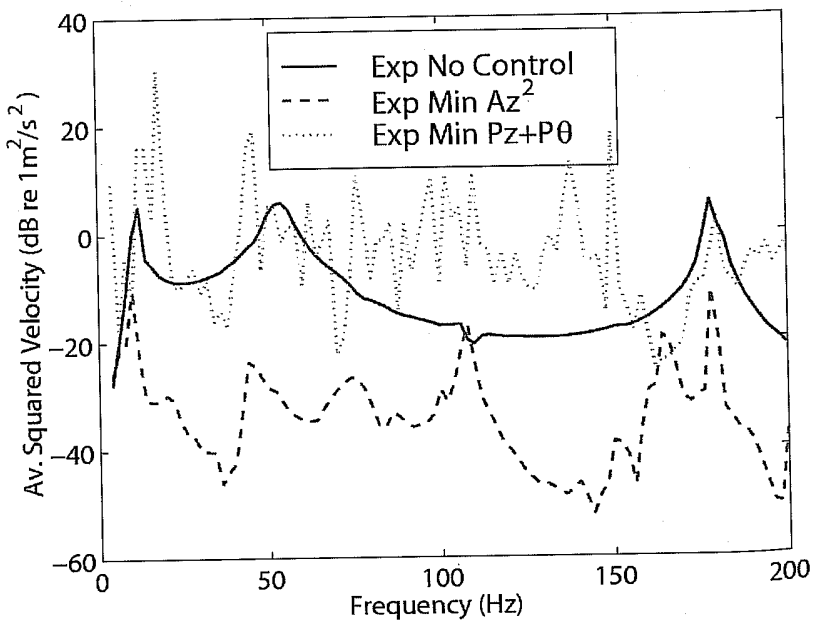

FIG. 16. (Color online) Experimental results of the average of the squared velocity of the beam for no control, minimization of $A_{z}^{2}$ and minimization of the sum of the signed power transmission $P_{z}$ and $P_{\theta y}$. 


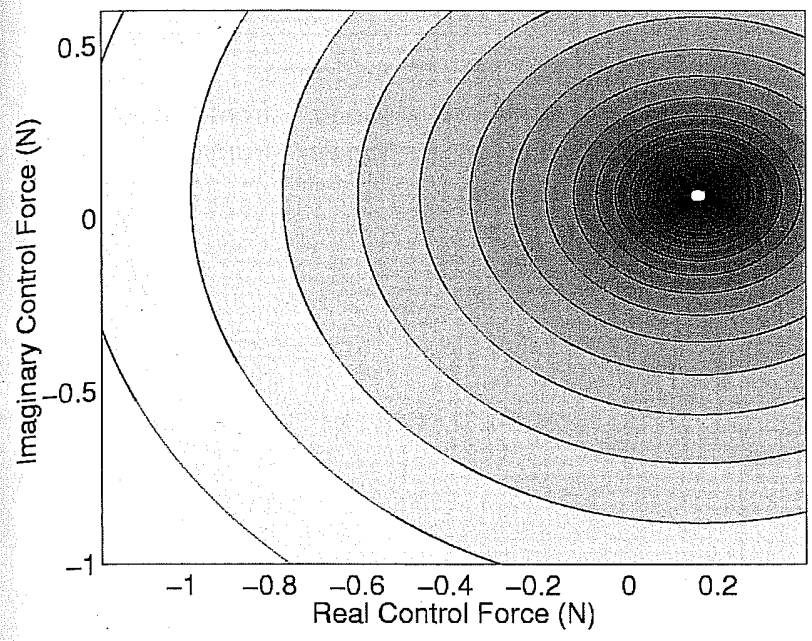

FIG. 17. Contour plot of the theoretical squared power transmission $P_{z}^{2}$ $+P_{\theta y}^{2}$ along the vertical and rotational axes with no phase error. The white dot shows the control force which minimizes the squared total power transmission. Power transmission is inversely proportional to the darkness of the contour.

\section{Squared power transmission}

The results from Howard ${ }^{9}$ show that the minimization of squared power transmission gives results better than the minimization of signed power transmission when negative values of signed power transmission are possible, even though random phase errors also cause an error in the measurement of squared power transmission. The theoretical model can be used to show the effect of phase errors on the isolation performance for the minimization of squared power transmission. Figure 17 shows a contour plot of the squared total power transmission at $100 \mathrm{~Hz}$ for the theoretical model when transducers have no phase errors. The shading indicates constant levels of squared power transmission, and darker shading indicates values that are closer to zero. The axes are the real and imaginary parts of the control force, and the white dot at the center of the rings corresponds to the value of the control force which minimizes the squared total power transmission. This result shows that if the transducers had no phase errors, then the error surface would resemble a parabolic bowl. If the transducers have phase errors then the error surface will not have a unique global minimum but will have an infinite number of solutions for the control force which will minimize the erroneous measure of squared power transmission, as shown in Fig. 18 as the dark ring. Figure 18 shows a white dot which is at the same location as the white dot in Fig. 17. This is the control force which an adaptive controller should converge towards. The error surface shown in Fig. 18 resembles a parabolic bowl with an inverted bowl at the center of the parabola. Figure 19 shows a close-up of Fig. 18 around the control force which minimizes the total power transmission with no phase error. Figure 19 shows that the control force which minimizes the true value of squared total power transmission does not lie on the ring of solutions which minimizes the erroneous measure of squared total power transmission. Obviously an adaptive controller should converge towards the true value, but the controller could converge to any solution on the dark ring shown in Fig. 18. The controller needs to be guided towards

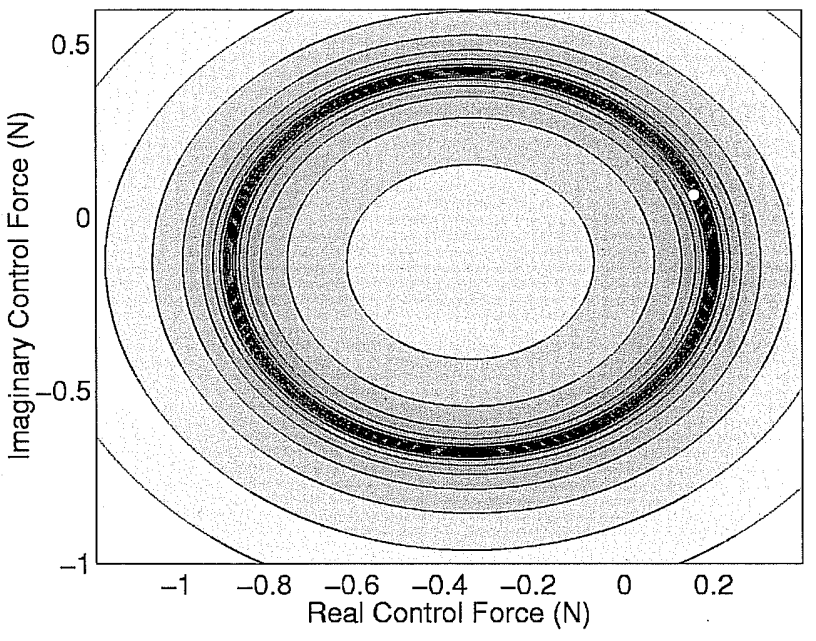

FIG. 18. Contour plot of the theoretical squared power transmission $P_{z}^{2}$ $+P_{\theta y}^{2}$ along the vertical and rotational axes with a $\pm 2^{\circ}$ phase error. The white dot shows the control force which minimizes the squared total power transmission with no phase error. Power transmission is inversely proportional to the darkness of the contour. Minimum power transmission is represented by the black ring.

the true value. Figure 19 also shows the control force which minimizes the squared acceleration along the vertical $Z$ axis, which has a value near to the control force which minimizes the true value of squared total power transmission. An adaptive controller could be guided towards minimizing the squared acceleration, which will start the adaptation process in the correct direction towards minimizing the true value of squared power transmission. Once the controller had minimized the cost function of squared acceleration, the cost function was altered so that it minimized the squared power transmission. This technique was used here and the control force which was calculated is shown in Fig. 19 as a white dot which lies on the ring of solutions where the squared total power transmission (with phase errors) equals zero. These same three solutions for the control force are shown in Fig.

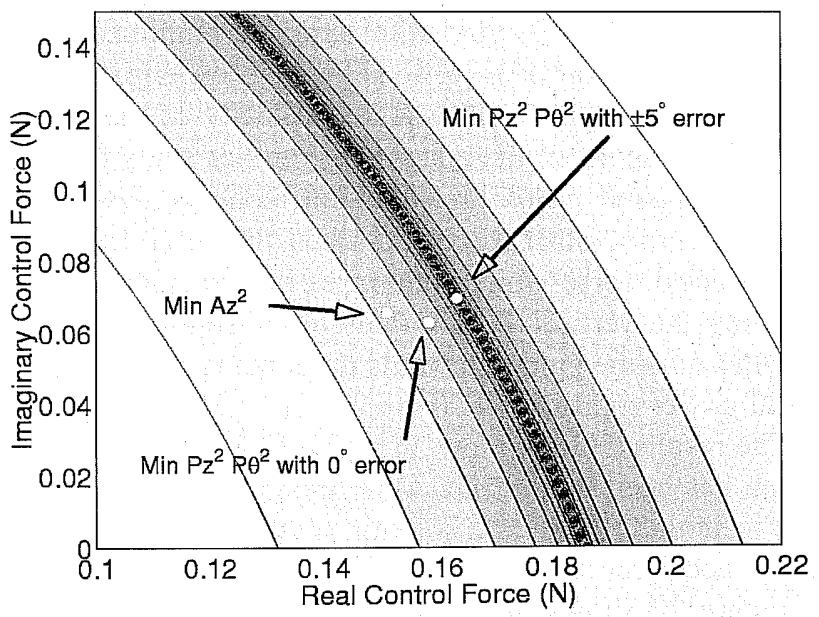

FIG. 19. Contour plot of the theoretical squared power transmission $P_{z}^{2}$ $+P_{\theta y}^{2}$ along the vertical and rotational axes with a $\pm 2^{\circ}$ phase error showing the three different control forces which minimize the squared acceleration along the vertical axis, the squared total power transmission with no phase error, and the squared total power transmission with $\pm 2^{\circ}$ phase error. Power transmission is inversely proportional to the darkness of the contour. Minimum power transmission is represented by the black ring. 


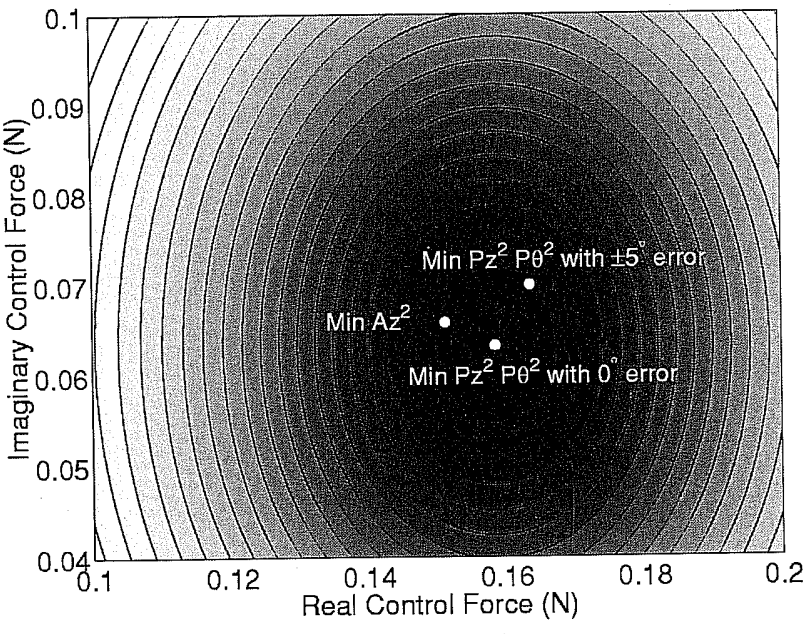

FIG. 20. Contour plot of the theoretical squared power transmission $P_{z}^{2}$ $+P_{\theta y}^{2}$, along the vertical and rotational axes with no phase error, showing the three different control forces which minimize, respectively, the squared acceleration along the vertical axis, the squared total power transmission with no phase error, and the squared total power transmission with $\pm 2^{\circ}$ phase error. Power transmission is inversely proportional to the darkness of the contour.

20 , where the contours show the true value of total power transmission, that is with no phase error. The control force which is closest to the control force which minimizes the true value of the squared total power transmission is the better solution. In this case the control force which minimizes the squared acceleration along the vertical axis and the control force which minimizes the squared total power transmission with phase errors have about the same value of total power transmission as they are both on the same contour level.

It is not possible to experimentally demonstrate this phenomenon as the force transducers and accelerometers used in the experiments have phase errors and cannot be compared with an experiment without phase errors. It is possible to experimentally demonstrate the technique described above where the adaptation is guided towards the minimization of squared acceleration. Figure 21 shows the experimental results for the average of the squared velocity of the simply supported beam when the adaptive controller starts to minimize the squared power transmission along the vertical $Z$ axis from zero control force and when the controller starts from a control force which minimizes the squared acceleration along the vertical $Z$ axis. This result confirms that the controller must be guided towards minimizing the true value of total power transmission (with no phase error).

The results which follow, in which squared power transmission has been minimized, were obtained using this technique to initially guide the solution towards minimizing the squared acceleration.

Figure 22 shows the theoretically predicted average of the squared velocity of the beam for no control, when the squared power transmission $P_{z}^{2}$ along the vertical axis is minimized, and when the sum of the squared power transmission $P_{z}^{2}+P_{\theta y}^{2}$ along the vertical and rotational axes is minimized when there is a random $\pm 2^{\circ}$ phase error. This result shows that phase errors associated with the measure-

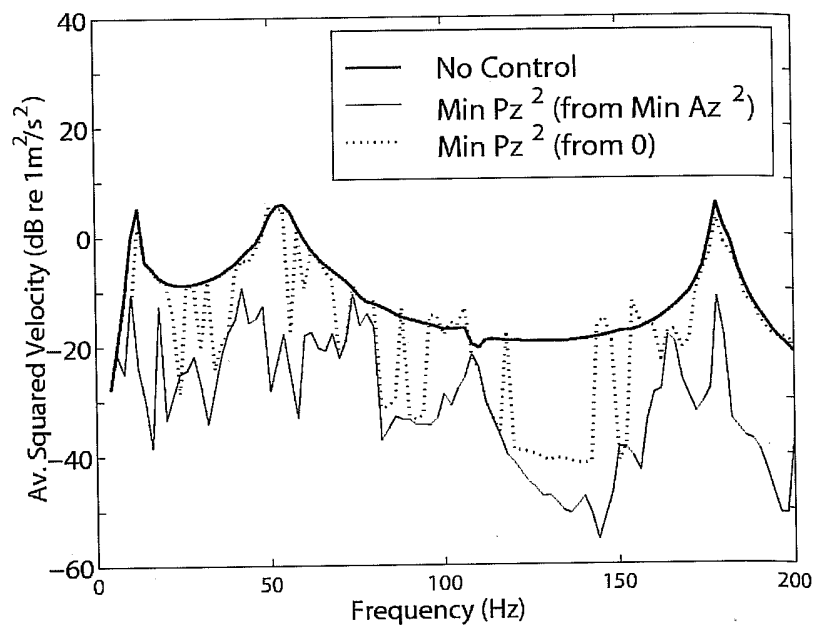

FIG. 21. Experimentally measured average of the squared velocity of the beam when the adaptive controller starts to minimize the squared power transmission along the vertical axis from zero control force and when the adaptation starts from the minimization of squared acceleration $A_{z}^{2}$.

ment of power will not greatly affect the minimization of squared power transmission. This prediction was confirmed by experiment as shown in Fig. 23. It can be seen that the minimization of squared power transmission along the vertical and rotational axes results in a lower average of the squared velocity of the beam at the rotational resonance of $108 \mathrm{~Hz}$.

Another experiment was conducted for the case where the rigid mass was excited along both the vertical axis and the horizontal axis aligned with the beam. The results from these experiments are not presented as they are similar to those described above, except that the peak corresponding to the rotational resonance at $108 \mathrm{~Hz}$ is larger for both the controlled and uncontrolled cases.

Figure 24 shows that the cost functions considered so far provide similar levels of vibration isolation. However, the greatest vibration isolation obtained by the minimization of

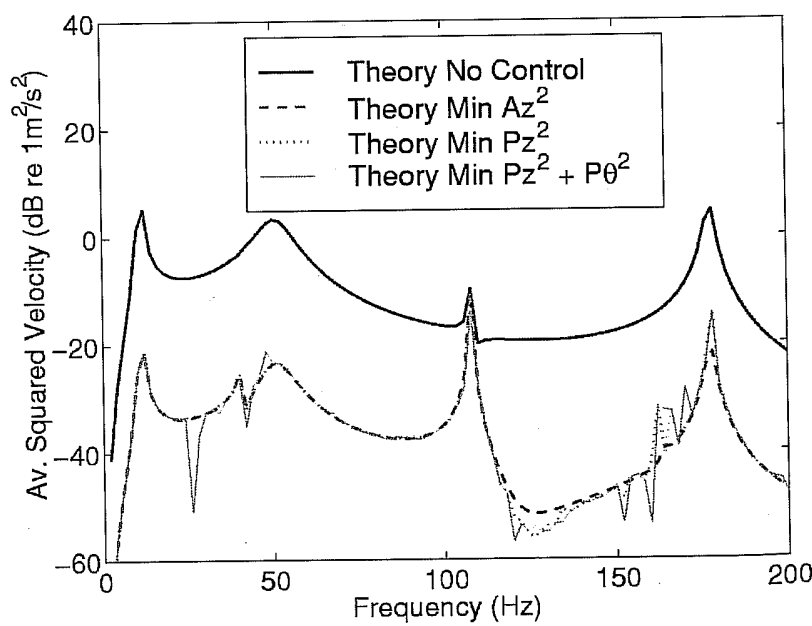

FIG. 22. (Color online) Theoretical prediction of the average of the squared velocity of the beam for no control, minimization of squared acceleration $A_{3}^{2}$ along the vertical axis, minimization of squared power transmission $P_{z}^{2}$ along the vertical axis with a random $\pm 2^{\circ}$ phase error and the minimization of the sum of the squared power transmissions $P_{z}^{2}+P_{\theta y}^{2}$ along the vertical and rotational axes with a random $\pm 2^{\circ}$ phase error. 


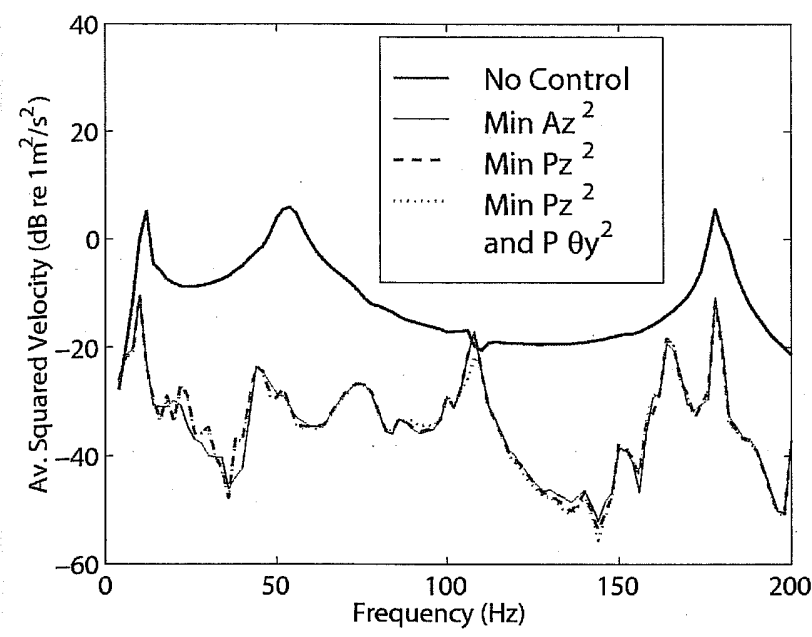

FIG. 23. (Color online) Experimental results of the average of the squared velocity of the beam for no control, minimization of squared acceleration $A_{z}^{2}$ along the vertical axis, minimization of squared power transmission $P_{z}^{2}$ along the vertical axis and the minimization of the sum of the squared power transmissions $P_{z}^{2}+P_{\theta y}^{2}$ along the vertical and rotational axes.

the weighted sum of squared velocity and force along translational and rotational axes was slightly better than that obtained using the other cost functions.

\section{CONCLUSIONS}

A novel transducer was used to investigate the effectiveness of various cost functions for actively minimizing the transmission of vibration from a vibrating rigid mass to a simply supported beam. The active isolator was intended to control vibration transmission only along the vertical axis, and the transducer was used as an error sensor allowing minimization of vibration along any translational or rotational axis or combination of axes. The effectiveness of each

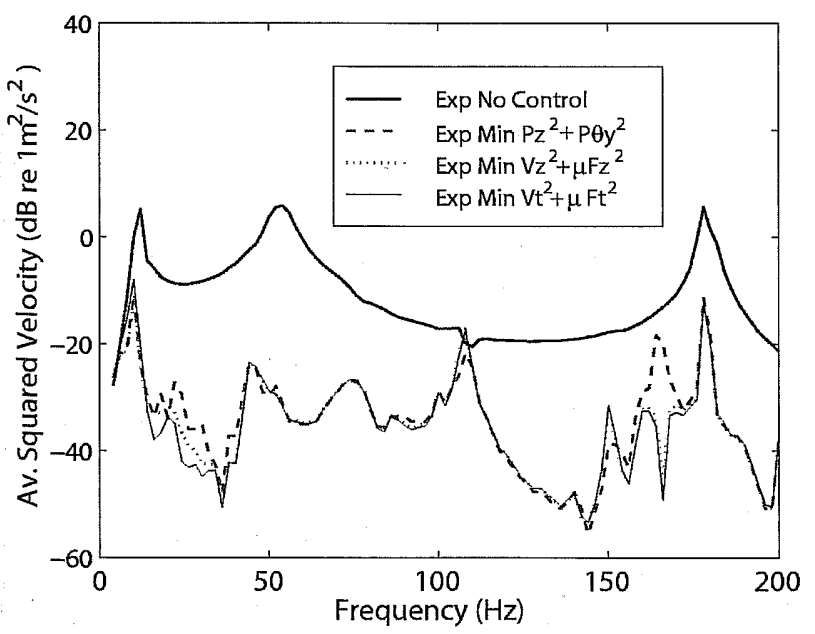

FIG. 24. (Color online) Experimental results of the average of the squared velocity of the beam for no control, minimization of squared acceleration $A_{z}^{2}$ along the vertical axis, minimization of the sum of the squared power transmissions $P_{z}^{2}+P_{\theta y}^{2}$ along the vertical and rotational axes, minimization of the weighted sum of $V_{z}^{2}+\mu F_{z}^{2}$, the squared velocity and force along the vertical axis and the minimization of $V_{t}^{2}+\mu F_{z}^{2}$, the weighted sum of squared velocities, forces, and moments along translational and rotational axes. cost function was evaluated by measuring the vibration levels in the simply supported beam which acted as the receiving structure.

The experimental results showed that the minimization of the signed value of power transmission was ineffective in minimizing the vibration transmitted into the simply supported beam, which was due to insufficient phase accuracy of the transducer used to derive the signed power transmission for the error signal for the controller. Theoretical predictions were made which included random phase errors for the error transducer, and these predicted results were comparable to the measured experimental results. The results obtained by minimizing the squared value of power transmission were an improvement over those obtained by minimizing the signed value of power transmission. However, the phase accuracy of the transducer still limited the maximum vibration attenuation that could be achieved. Although the measurement of total vibrational power transmission is theoretically appealing because the vibrational energy from the contribution of translational and rotational vibration uses consistent units of watts, this metric will unfortunately always be limited by the phase accuracies of the transducers used to determine power transmission.

The best vibration isolation performance was obtained from the minimization of the weighted sum of the squared translational forces and velocities and squared rotational moments and velocities. This cost function is not limited by the phase accuracies of the transducers and hence it is more practical than the measurement of vibrational power transmission. Similar results have been shown for the acoustic equivalent where energy density sensing has been shown to be more practical than the minimization of sound intensity. ${ }^{35}$ The combined force, moment, and velocity signals had to be weighted appropriately so that the amplitude of each signal was similar, so as not to favor the attenuation of one vibration along or around one axis over that corresponding to another axis. The appropriate weighting factor is a function of the structural impedance measured at the error transducer.

It can be justifiably claimed that the adjustment of the signal amplitudes constitutes the creation of an artificially optimum cost function, whereas the cost function of the total vibrational power transmission is independent of the system configuration under investigation. Although there is elegance in the academic power transmission approach, the use of squared force and velocity signals is a realizable and much more practical solution.

${ }^{1}$ C. Q. Howard, "Active isolation of machinery vibration from flexible structures," Ph.D. thesis, University of Adelaide, Australia, 1999.

${ }^{2}$ J. Q. Pan, C. H. Hansen, and J. Pan, "Active isolation of a vibration source from a thin beam using a single active mount," in Proceedings of InterNoise '91 (INCE, Sydney, Australia, 1991), pp. 683-686.

${ }^{3}$ J. Q. Pan, C. H. Hansen, and J. Pan, "Active isolation of a vibration source from a thin beam using a single active mount," J. Acoust. Soc. Am. 94(3), 1425-1434 (1993).

${ }^{4}$ J. Q. Pan and C. H. Hansen, "Active control of vibratory power flow from a vibrating rigid body to a flexible panel through two active isolators," $\mathrm{J}$. Acoust. Soc. Am. 93, 1947-1953 (1993).

${ }^{5}$ T. Royston and R. Singh, "Optimization of passive and active non-linear vibration mounting systems based on vibratory power transmission," J. Sound Vib. 194(3), 295-316 (1996).

${ }^{6} \mathrm{R}$. J. Pinnington, "The measurement of vibrational power input to a struc- 
ture by a multipole expansion," in Structural Intensity and Vibrational Energy Flow-3rd International Congress on Intensity Techniques (CETIM, Senlis, France, 1990), pp. 441-448.

${ }^{7}$ P. Gardonio, S. J. Elliot, and R. J. Pinnington, "Active isolation of structural vibration on a multiple degree of freedom system. I. The dynamics of the system," J. Sound Vib. 207(1), 61-93 (1997).

${ }^{8}$ P. Gardonio, S. J. Elliot, and R. J. Pinnington, "Active isolation of structural vibration on a multiple degree of freedom system. II. Effectiveness of active control strategies," J. Sound Vib. 207(1), 95-121 (1997).

${ }^{9}$ C. Q. Howard and C. H. Hansen, "Finite element analysis of active vibration isolation using vibrational power as a cost function," Int. J. Acoust. Vib. 4(1), 23-36 (1999).

${ }^{10} \mathrm{~B}$. Gibbs and S. Yap, "The contribution of forces and moments to the structure-borne sound power from machines rigidly attached to supporting floors," in Proceedings of Inter-Noise '98 (INCE, Christchurch, New Zealand, 1998).

${ }^{11}$ M. D. Jenkins, "Active control of periodic machinery vibrations," Ph.D. thesis, University of Southampton, 1989.

${ }^{12}$ S. D. Sommerfeldt, "Multi-channel adaptive control of structural vibration," Noise Control Eng. J. 37(2), 77-89 (1991).

${ }^{13}$ B. A. T. Petersson and B. M. Gibbs, "The influence of source location with respect to vibrational energy transmission," in 3rd International Congress on Intensity Techniques (CETIM, Senlis, France, 1990), pp. 449-456.

${ }^{14}$ B. A. T. Petersson, "Moment and force excitation at edges and corners of beam and plate like structures," in International Conference on Recent Advances in Structural Dynamics (ISVR, Southampton, 1991), pp. 148157.

${ }^{15}$ Y. K. Koh and R. G. White, "Analysis and control of vibrational power transmission to machinery supporting structures subjected to a multiexcitation system. I. Driving point mobility matrix of beams and rectangular plates," J. Sound Vib. 196(4), 469-493 (1997).

${ }^{16}$ Y. K. Koh and R. G. White, "Analysis and control of vibrational power transmission to machinery supporting structures subjected to a multiexcitation system. II. Vibrational power analysis and control schemes," J. Sound Vib. 196(4), 495-508 (1997).

${ }^{17}$ Y. K. Koh and R. G. White, "Analysis and control of vibrational power transmission to machinery supporting structures subjected to a multiexcitation system. III. Vibrational power cancellation and control experiments," J. Sound Vib. 196(4), 509-522 (1997).

${ }^{18}$ M. Sanderson, "Vibration isolation: Moments and rotations included," J. Sound Vib. 198, 171-191 (1996).

${ }^{19}$ A. Moorhouse, "A dimensionless mobility formulation for evaluation of force and moment excitation," J. Acoust. Soc. Am. 112(3), 972-980 (2002).

${ }^{20} \mathrm{~L}$. Ji, B. Mace, and R. Pinnington, "A power mode approach to estimating vibrational power transmitted by multiple moments," J. Sound Vib. 265 ,
387-399 (2003).

${ }^{21}$ T. J. Royston and R. Singh, "Optimization of passive and active non-linear vibration mounting systems based on vibratory power transmission," J. Sound Vib. 194(3), 295-316 (1996).

${ }^{22}$ K. M. Misovec, F. F. Flynn, B. G. Johnson, and J. K. Hedrick, "Sliding mode control of magnetic suspensions for precision pointing and tracking applications," in American Society of Mechanical Engineers, Winter Annual Meeting - Active Noise and Vibration Control (ASME, Dallas, TX, 1990), Vol. NCA - 8, pp. 75-82.

${ }^{23}$ R. C. Fenn, J. R. Downer, V. Gondhalekar, and B. G. Johnson, "An active magnetic suspension for space-based microgravity vibration isolation," in American Society of Mechanical Engineers, Winter Annual Meeting - Active Noise and Vibration Control (ASME, Dallas, TX, 1990), Vol. NCA 8, pp. 49-56.

${ }^{24}$ C. H. Gerhold and R. Rocha, "Active vibration control in microgravity environment," Trans. ASME, J. Vib., Acoust., Stress, Reliab. Des. 110, 30-35 (1988).

${ }^{25}$ D. A. Kienholz, "Defying gravity with active test article suspension systems," Sound Vib., 14, 14-21 (1994).

${ }^{26}$ C. Ross, J. Scott, and S. Sutcliffe, Active Control of Vibration, International Patent Application No. PCT/GB87/00902, 1988.

${ }^{27}$ C. Ross, J. Scott, and S. Sutcliffe, Active Control of Vibration, US Patent No. 5,433,422, 1989.

${ }^{28}$ S. Sutcliffe, G. Eatwell, and S. Hutchins, Active Control of Vibration, UK Patent No. GB 2,222,657, 1989.

${ }^{29}$ M. A. Sanderson, "Direct measurement of moment mobility. II. An experimental study," J. Sound Vib. 179(4), 685-696 (1995).

${ }^{30}$ C. Dorling, G. Eatwell, S. Hutchins, C. Ross, and S. Sutcliff, "A demonstration of active noise reduction in an aircraft," J. Sound Vib. 112(2), 389-395 (1987).

${ }^{31}$ C. Dorling, B. Eatwell, S. Hutchins, C. Ross, and S. Sutcliffe, "A demonstration of active noise reduction in an aircraft cabin," J. Sound Vib. 128(2), 358-360 (1989).

${ }^{32}$ P. A. Nelson and S. J. Elliott, Active Control of Sound (Academic, San Diego, 1992).

${ }^{33} \mathrm{G}$. Pavić, "On the relationship between the energy flow into a structure and its vibration," in Structural Intensity and Vibrational Energy Flow 4th International Congress on Intensity Techniques (CETIM, Senlis, France, 1993), pp. 95-99.

${ }^{34} \mathrm{E}$. Henriksen, "Adaptive active control of structural vibration by minimisation of total supplied power," in Inter-Noise 96 (INCE, Liverpool, England, 1996), pp. 1615-1618.

${ }^{35}$ Y. Park and S. Sommerfeldt, "Global attenuation of broadband noise fields using energy density control," J. Acoust. Soc. Am. 101(1), 350-359 (1997). 